\title{
Triangle tilt surgery as salvage procedure for failed shoulder surgery in obstetric brachial plexus injury
}

\author{
Rahul K. Nath $\cdot$ Meera B. Avila $\cdot$ Priyanka Karicherla
}

Accepted: 15 July 2010/Published online: 29 July 2010

(C) The Author(s) 2010. This article is published with open access at Springerlink.com

\begin{abstract}
Purpose The study was conducted to review the effects of triangle tilt surgery in children with OBPI (obstetric brachial plexus injury) who had previously undergone several operative procedures at other hospitals before presenting at our institute.

Methods The study included a group of 48 OBPI patients who had undergone previous operative procedures at outside hospitals by other surgeons. Patients were assessed for shoulder function using their radiological reports and the modified Mallet functional scale. The same patients underwent the triangle tilt procedure at our institution and were re-evaluated for shoulder function.

Results The results of the study showed an increase in Mallet scores from 11.88 points to 15.17 points $(p<0.01)$, improvement in PHHA (percentage of humeral head anterior to the glenoid) from $14 \%$ to $25 \%(p<0.05)$, enhancement in glenoid version from $-32^{\circ}$ to $-25^{\circ}$ $(p<0.01)$, and a decrease in the SHEAR (scapular hypoplasia, elevation, and rotation) deformity after surgery.

Conclusion The data obtained demonstrated that the triangle tilt procedure significantly enhanced shoulder function and glenohumeral congruity in these patients as evidenced by the improvements in Mallet scores, PHHA, glenoid version, and SHEAR deformity.
\end{abstract}

\footnotetext{
R. K. Nath $(\bowtie) \cdot$ M. B. Avila · P. Karicherla

Texas Nerve and Paralysis Institute, 6400 Fannin Street,

Suite 2420, Houston, TX 77030, USA

e-mail: drnath@drnathmedical.com

M. B. Avila

e-mail: meera@drnathmedical.com

P. Karicherla

e-mail: priyanka@drnathmedical.com
}

Keywords Obstetric brachial plexus injury · Glenohumeral congruity · Shoulder function . Triangle tilt surgery

\section{Introduction}

Obstetric brachial plexus injury (OBPI) is a nerve injury that affects the brachial plexus at childbirth [1]. The most commonly affected roots are C5-6 (Erb's palsy), forming the upper trunk of the plexus. Less frequently, the entire plexus (C5-T1) may be affected. OBPI appears to have an incidence of 0.4-4.6 injuries every 1000 live births [2]. A significant proportion of patients suffer from limb dysfunction and permanent bony changes at the shoulder. Because the brachial plexus injury tends to be asymmetric, it will lead to muscle imbalances about the shoulder. These imbalances lead to glenohumeral dysplasia and joint incongruity [3]. The major bony and joint problem that occurs is known as the SHEAR (scapular hypoplasia, elevation, and rotation) deformity, which is recognized clinically as twisting of the scapula above the level of the clavicle [4]. The abnormal anterior rotation of the clavicle along with the elevated scapula causes the acromioclavicular plane to tilt anteriorly and among other things, causes impingement of the acromion upon the humeral head. This restricts external rotation of the shoulder and contributes to anterior glenohumeral tightness of the soft tissues $[3,5]$. Other significant secondary deformities that follow include medial rotation contracture of the shoulder and elbow flexion contracture.

The muscular instability about the shoulder girdle leads to glenohumeral dysplasia and joint incongruity $[3,4]$. The major bony deformity that develops is termed as the SHEAR (scapular hypoplasia, elevation, and rotation) 
deformity, which is caused by the elevation and extrusion of the affected scapula beyond the clavicle [5]. The abnormal anterior rotation of the clavicle together with the protracted scapula causes the acromioclavicular plane to tilt forward and thereby lead to the impingement of the acromion upon the humeral head [3, 6]. Significant secondary deformities that follow include medial rotation contracture (MRC) and elbow flexion.

Management of significant shoulder deformities in OBPI has typically been through the performance of various operative procedures including axillary nerve decompression, humeral osteotomy, biceps tendon lengthening, glenohumeral capsulorrhaphy and anterior capsule release [7-13]. There are no peer-reviewed studies documenting functional improvements in OBPI patients through the use of therapy alone. Although humeral osteotomy may improve the cosmesis of the arm at rest and shoulder external rotation to some degree, it does not correct, attempt to correct or address glenohumeral dysplasia; therefore, it is at best an incomplete and simplistic solution for a complex problem. Humeral osteotomy does not stimulate shoulder joint remodeling. Anterior capsule release alone often causes the humerus to align in an extreme externally rotated position and thereby results in loss of internal rotation, leading to a deformity worse than that of the initial injury. Surgical procedures such as glenohumeral capsulorrhaphy, humeral osteotomy, anterior capsule release, and nerve transfers also do not attempt to correct the SHEAR deformity, and have minimal or adverse effects on the glenohumeral anatomic derangements. The triangle tilt surgery was therefore developed by the senior author to directly address the impingement of the acromion on the humeral head, so as to allow for the repositioning of the humeral head into the glenoid and to stimulate favorable joint remodeling. The surgical technique includes osteotomies of the clavicle and the neck of the acromion, allowing the distal acromioclavicular triangle to tilt back to a more neutral position and allowing the humeral head to improve congruency [14-16].

The current study was conducted to review the effects of triangle tilt surgery in children with OBPI who had previously undergone several types of operative procedures at other hospitals (posterior glenohumeral capsulorrhaphy, biceps tendon lengthening, forearm osteotomy, humeral osteotomy, or anterior capsule release) before presenting at our institute. These previous surgeries were intended to improve shoulder function but did not accomplish this fully or at all in all cases in the current study. The results from this study show that the triangle tilt surgery was able to improve the shoulder function in all patients who had previously undergone unsuccessful operative procedures to correct the deformity.

\section{Patients and methods}

\section{Patients}

A retrospective study was conducted on 48 OBPI patients who had had prior operative procedures at another institute to correct shoulder deformities before presentation at this institute. The patient population consisted of 25 males and 23 females of whom 18 patients had left-sided injuries and 30 had right-sided injuries. The nerve involvement was $\mathrm{C} 5$, $6(N=27) ; \mathrm{C} 5,6,7(N=13)$ or C5, 6, 7, 8, T1 $(N=8)$. Prior operative procedures that the patients underwent at another center included posterior glenohumeral capsulorrhaphy $(N=20)$, biceps tendon lengthening $(N=19)$, forearm osteotomy $(N=2)$, humeral osteotomy $(N=14)$, and anterior capsule release $(N=4)$. All patients underwent the modified Quad (MQ) procedure at this institute prior to the triangle tilt procedure in order to improve global abduction. The functional benefits of MQ have been discussed in a previous work by the senior author [16].

\section{Radiological evaluation}

In order to assess bony deformities of the shoulder joint, CT or MRI images of the patients were studied before and after triangle tilt surgery. Posterior humeral head subluxation, glenoid version, and SHEAR deformity were measured from the radiographs (CT/MRI scans) by trained research scientists independent of the surgeon and senior author.

Glenoid version (normal value $=0$ ) was measured as described by Friedman et al. [17] using axial CT/MRI images. A scapular line connecting the mid-glenoid to the medial spine of the scapula was constructed using Universal Desktop Ruler (AVPSoft.com, Voronezh, Russia). The angle formed between the scapular line and a line drawn tangential to the glenoid surface interacting closely with the humeral head was calculated and $90^{\circ}$ was subtracted from it to measure the glenoscapular angle.

Posterior subluxation of the humeral head (Fig. 1), expressed as percentage of humeral head anterior to the glenoid (PHHA, normal value $=50$ ), was calculated from the ratio of the distance between the scapular line to the anterior aspect of humeral head and the greatest diameter of the head multiplied by 100 .

The scapular deformity, also referred to as SHEAR deformity, was measured from the 3D reconstructions of the CT images. The area of the scapula visible above the clavicle was measured and divided with the total area of the scapula for both affected and normal sides. The ratio of the affected side was subtracted from that of the normal side and multiplied with 100 to obtain SHEAR deformity (normal value $=0$ ). 
Fig. 1 Axial computerized tomographic (CT) scans of the affected shoulder of an OBPI patient before and after triangle tilt surgery. a CT image before triangle tilt surgery showing posterior subluxation of the humeral head, measured by PHHA, and joint incongruity of the glenohumeral joint, measured by glenoid version. b Line diagram of the pretriangle tilt CT image (a). c CT image after triangle tilt surgery showing improvement in PHHA and glenoid version. $\mathbf{d}$ Line diagram of the post-triangle tilt $\mathrm{CT}$ image (c) (Figure was prepared using the computer program Photoshop)
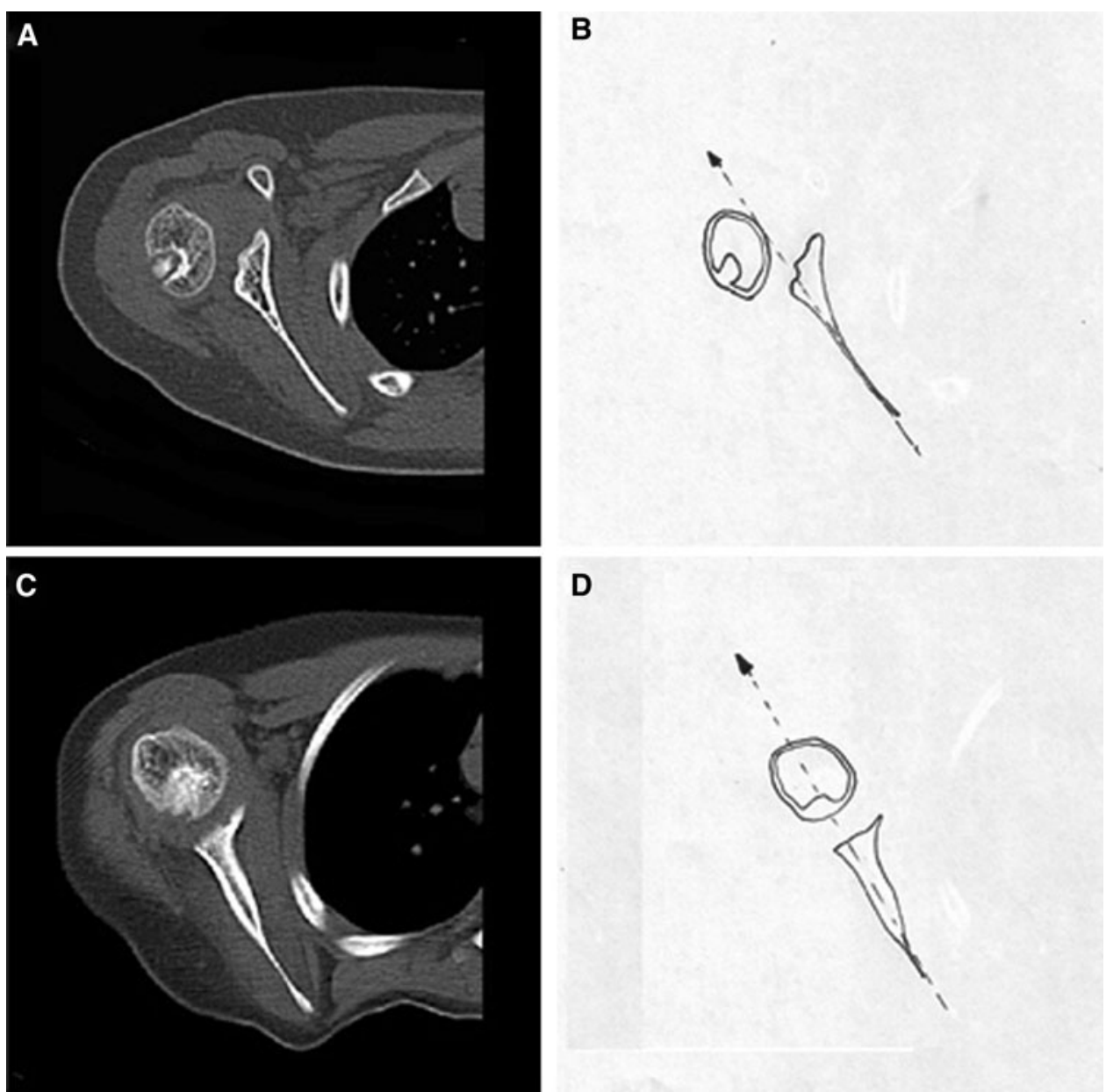

D
Clinical assessment

Shoulder function was assessed through the modified Mallet scale through video recordings of patients performing the following movements: external rotation, hands to mouth, hands to neck, hands to spine, and supination. For each functional Mallet parameter, patients were scored on a scale of $1-5$ with 5 as normal function and 1 denoting lack of any movement.

Operative technique

The triangle tilt surgical procedure developed by the lead author was shown to have successful outcomes in OBPI patients $[15,18]$. The operative procedure includes clavicle osteotomy at the intersection of its middle and distal third, acromion osteotomy at its intersection with the scapular spine and osteotomy of the scapula followed by splinting of the limb in adduction [15]. All surgical procedures were performed by the senior author who has over 14 years of experience in this field with several thousand OBPI patients.
Statistical analysis

The paired Student's $t$ test statistic was applied to compare the mean Mallet scores and bony parameters between the pre- and post-triangle tilt groups using the 'Analyse it' plugin (Leeds, UK) for Microsoft Excel 2003. A value of $p<0.05$ was considered to be statistically significant.

\section{Results}

Patient demographics are shown in Table 1. The outcome of triangle tilt surgery in OBPI patients is shown in Tables 2, 3 and Figs. 1, 2. All patients who had presented with severe shoulder deformities had undergone operative procedures at another hospital before presenting at our institute. At their latest clinic visit (mean followup $=42$ months), the patients displayed a significant improvement in the mean Mallet score from 11.88 points preoperatively to 15.17 points postoperation (Table 2, $p<0.01)$. Improvements were noticed in all the functional Mallet parameters including external rotation $(p<0.0001)$, 
Table 1 Patient demographics

\begin{tabular}{lc}
\hline Patient gender & Number of patients \\
\hline Male & 25 \\
Female & 23 \\
Side of injury & \\
Left & 18 \\
Right & 30 \\
Operations at another institutes & \\
PGHC & 30 \\
BTL & 19 \\
FO & 2 \\
HO & 14 \\
ACR & 4 \\
Total & 69 \\
Nerve involvement & \\
C5, C6 & 27 \\
C5, C6, C7 & 13 \\
C5, C6, C7, C8 (T1) & 8 \\
\hline
\end{tabular}

$P G H C$ posterior glenohumeral capsulorrhaphy, BTL biceps tendon lengthening, $F O$ forearm osteotomy, $H O$ humeral osteotomy, $A C R$ anterior capsule release

Table 2 Comparison of Mallet scores before and after triangle tilt surgery

\begin{tabular}{lccl}
\hline & Pre $\mathrm{TT}^{\mathrm{a}}$ & Post $^{\mathrm{b}}$ & $p$ Value \\
\hline External rotation & $2.29 \pm 0.07$ & $3.1 \pm 0.12$ & $<0.0001$ \\
Hand-to-mouth & $1.98 \pm 0.91$ & $3.04 \pm 0.92$ & $<0.0001$ \\
Hand-to-neck & $2.67 \pm 0.1$ & $3.2 \pm 0.1$ & $<0.0001$ \\
Hand-to-spine & $2.29 \pm 0.6$ & $2.54 \pm 0.1$ & $<0.01$ \\
Supination & $2.65 \pm 0.89$ & $3.29 \pm 0.68$ & $<0.0001$ \\
Total Mallet score & $11.88 \pm 0.29$ & $15.17 \pm 0.29$ & $<0.01$ \\
\hline
\end{tabular}

${ }^{a}$ Pre-TT: before triangle tilt surgery

b Post-TT: after triangle tilt surgery

Table 3 Comparison of bony parameters before and after triangle tilt surgery

\begin{tabular}{lrrr}
\hline & Pre-TT $^{\mathrm{a}}$ & Post-TT $^{\mathrm{b}}$ & $p$ Value \\
\hline Posterior subluxation $(\%)$ & $14 \pm 3$ & $25 \pm 3$ & $<0.05$ \\
Glenoid version $\left({ }^{\circ}\right)$ & $-32 \pm 3$ & $-23 \pm 3$ & $<0.001$ \\
\hline
\end{tabular}

${ }^{\text {a }}$ Pre-TT: before triangle tilt surgery

b Post-TT: after triangle tilt surgery

hands to mouth $(p<0.0001)$, hands to neck $(p<0.0001)$, hands to spine $(p<0.01)$, and forearm supination $(p<0.0001)$. The position of the arm at rest also improved following surgery (Fig. 2). All patients had undergone a modified Quad surgery at this institution prior to the triangle tilt procedure to improve global abduction. Therefore, this parameter has not been presented in the current study. In the current study, therefore, all preoperative measurements were taken prior to triangle tilt surgery but after modified Quad surgery had already been done.

In addition to functional improvements, anatomical improvements were also noticed following surgery. Posterior subluxation (Fig. 1) measured using the PHHA value, improved from $14 \%$ preoperatively to $25 \%$ postoperatively $(p<0.05)$. There was also significant improvement in glenoid retroversion from $-32^{\circ}$ preoperatively to $-23^{\circ}$ postoperation $(p<0.001)$. The scapulae were less elevated after the surgery, thereby decreasing the SHEAR deformity. Taken together, this study demonstrates that the triangle tilt surgery enhanced shoulder function and anatomy in OBPI patients with prior failed surgeries.

\section{Discussion}

OBPI is a significant nerve injury that ultimately involves muscular instability and glenohumeral dysplasia that subsequently severely affects shoulder development and function. Restoration of glenohumeral congruity is therefore a primary objective in treating OBPI, which then allows for maximum functional range of motion and improved limb growth.

Many secondary surgical procedures have been described to manage the developmental derangements of OBPI. Traditionally, muscle releases and tendon transfers have been used to treat this deformity. Although these procedures can improve function and stop the exacerbation of glenohumeral dysplasia, they have not been shown to stimulate joint remodeling [9]. A recent study by our institute showed that OBPI patients who underwent nerve reconstructions developed more serious shoulder complications and required more secondary surgical procedures to address these deformities [19]. Humeral osteotomy is commonly applied to treat OBPI and has been shown to improve shoulder position [20, 21]. However, this procedure does not address glenohumeral deformity and therefore does not address the major documented cause of long-term morbidity and pain in this group of patients. Operative procedures such as anterior capsule release, posterior glenohumeral capsulorrhaphy and forearm osteotomy have all been applied to OBPI $[10,22]$ but have failed to address the SHEAR deformity, which then allows for continued glenohumeral deformity and dysfunction.

The triangle tilt procedure was designed to address the SHEAR deformity and relieve the impingement caused by the acromion on the humeral head, and therefore to address clinical and anatomic situations that are not touched upon 
Fig. 2 Shoulder and arm functions in an OBPI patient before triangle tilt surgery but after humeral osteotomy by a highly experienced OBPI surgeon $(\mathbf{a}-\mathbf{c})$ and 21 months after triangle tilt surgery $(\mathbf{d}-\mathbf{f})$. Significant improvements were noticed in arm at rest, hand-tomouth, and supination positions of the patient's affected arm (right arm) (Figure was prepared using the computer program Photoshop)
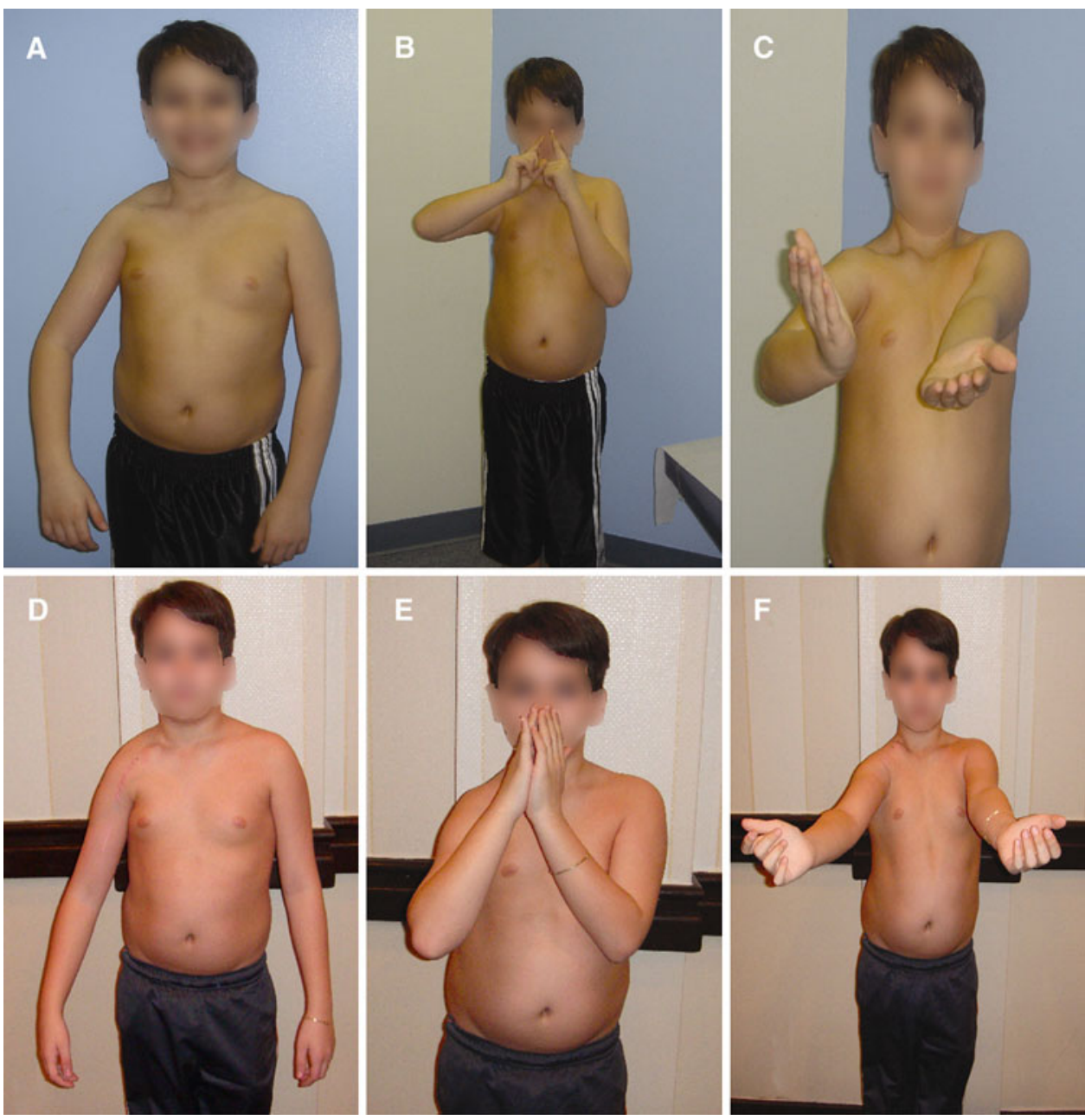

by previous operative procedures [15]. The procedure involves osteotomies of the clavicle, acromion, and osteotomy of the scapula. This allows for the acromioclavicular plane to return to a more neutral position, which then allows the humeral head to reposition itself into the glenoid in a more natural orientation. This aids in restoring glenohumeral congruity and permits maximum range of motion about the shoulder joint.

All patients in the current study were previously treated by surgeons at other hospitals and had undergone numerous surgical procedures (Table 1) prior to undergoing the triangle tilt procedure at our institute. The previous procedures either worsened the deformity or offered the patient limited functional assistance. The successful outcomes of the triangle tilt procedure are evidenced by the improvements in Mallet scores and glenohumeral joint alignment. The overall Mallet scores increased significantly ( $p<0.01$ ) from 11.88 to 15.17 points after triangle tilt surgery. Maximum improvements were seen in hands to mouth $(p<0.0001)$, hands to neck $(p<0.0001)$, and external rotation $(p<0.0001)$ functions. Considerable improvements were also seen in hands to spine $(p<0.01)$ function. The position of the arm at rest improved greatly (Fig. 2) as did forearm supination $(p<0.0001)$. Anatomical developments at the shoulder joint were also achieved through improvements in humeral head position, glenoid version and reduced SHEAR deformity.

The triangle tilt procedure is an effective surgical option for children with OBPI as it directly addresses and corrects the major shoulder bony deformity of OBPI. The surgery allows for overall improvements in shoulder function and glenohumeral congruity and anatomy, serving as a salvage procedure for patients who have had failed reconstructive procedures. This finding suggests that triangle tilt should be the initial standard of care for these patients, rather than reserved as a salvage procedure.

Acknowledgment The authors wish to thank Chandra Somasundaram for her revision of the manuscript.

Open Access This article is distributed under the terms of the Creative Commons Attribution Noncommercial License which permits any noncommercial use, distribution, and reproduction in any medium, provided the original author(s) and source are credited. 


\section{References}

1. Dunham EA (2003) Obstetrical brachial plexus palsy. Orthop Nurs 22(2):106-116

2. Maillet M, Romana C (2009) Complete obstetric brachial plexus palsy: surgical improvement to recover a functional hand. J Child Orthop 3:101-108

3. Nath RK, Humphries AD (2008) Computed tomography of the shoulders in patients with obstetric brachial plexus injuries: a retrospective study. Ann Surg Innov Res 2:4

4. Nath RK et al (2009) Triangle tilt surgery in an older pediatric patient with obstetric brachial plexus injury. ePlasty 9:e26

5. Nath RK, Paizi M (2007) Scapular deformity in obstetric brachial plexus palsy: a new finding. Surg Radiol Anat 29(2):133-140

6. Nath RK et al (2009) Arm rotated medially with supination - the ARMS variant: description of its surgical correction. BMC Musculoskelet Disord 10:32

7. Hoffer MM, Phipps GJ (1998) Closed reduction and tendon transfer for treatment of dislocation of the glenohumeral joint secondary to brachial plexus birth palsy. J Bone Joint Surg (Am) 80(7):997-1001

8. Safoury Y (2005) Muscle transfer for shoulder reconstruction in obstetrical brachial plexus lesions. Handchir Mikrochir Plast Chir 37(5):332-336

9. Waters PM, Bae DS (2005) Effect of tendon transfers and extraarticular soft-tissue balancing on glenohumeral development in brachial plexus birth palsy. J Bone Joint Surg (Am) 87(2):320-325

10. Kambhampati SB et al (2006) Posterior subluxation and dislocation of the shoulder in obstetric brachial plexus palsy. J Bone Joint Surg (Br) 88(2):213-219

11. Adelson PD, Nystrom NA, Sclabassi R (2005) Entrapment neuropathy contributing to dysfunction after birth brachial plexus injuries. J Pediatr Orthop 25(5):592-597

12. Rogers MH (1916) An operation for the correction of the deformity due to "obstetrical paralysis". Boston Med Surg J 174(5):163-164
13. Pearl ML et al (2006) Arthroscopic release and latissimus dorsi transfer for shoulder internal rotation contractures and glenohumeral deformity secondary to brachial plexus birth palsy. J Bone Joint Surg (Am) 88(3):564-574

14. Nath RK et al (2010) Long-term outcomes of triangle tilt surgery for obstetric brachial plexus injury. Pediatr Surg Int 26(4):393-399

15. Nath RK et al (2007) Surgical correction of the medial rotation contracture in obstetric brachial plexus palsy. J Bone Joint Surg (Br) 89(12):1638-1644

16. Nath RK, Paizi M (2007) Improvement in abduction of the shoulder after reconstructive soft-tissue procedures in obstetric brachial plexus palsy. J Bone Joint Surg (Br) 89(5):620-626

17. Friedman RJ, Hawthorne KB, Genez BM (1992) The use of computerized tomography in the measurement of glenoid version. J Bone Joint Surg (Am) 74(7):1032-1037

18. Nath RK, Melcher SE, Paizi M (2006) Surgical correction of unsuccessful derotational humeral osteotomy in obstetric brachial plexus palsy: Evidence of the significance of scapular deformity in the pathophysiology of the medial rotation contracture. J Brachial Plex Peripher Nerve Inj 1:9

19. Nath RK, Liu X (2009) Nerve reconstruction in patients with obstetric brachial plexus injury results in worsening of glenohumeral deformity: a case-control study of 75 patients. J Bone Joint Surg (Br) 91-B(5):649-654

20. Al-Qattan MM (2002) Rotation osteotomy of the humerus for Erb's palsy in children with humeral head deformity. J Hand Surg (Am) 27(3):479-483

21. Waters PM, Bae DS (2006) The effect of derotational humeral osteotomy on global shoulder function in brachial plexus birth palsy. J Bone Joint Surg (Am) 88(5):1035-1042

22. Pearl ML (2003) Arthroscopic release of shoulder contracture secondary to birth palsy: an early report on findings and surgical technique. Arthroscopy 19(6):577-582 